\title{
To Study the Pattern of Drug Resistance in Microbiologically Confirmed Cases of Tuberculosis and Risk Factors Associated with Drug Resistance Dr. Naveen Pandhi ${ }^{1}$, Dr. Ritu Dadra ${ }^{2 *}$, Dr. Balbir Malhotra ${ }^{1}$, Dr. P. Prasanth ${ }^{2}$
}

${ }^{1}$ Professor, Department of Chest and TB, Government Medical College, Amritsar, Punjab 143001, India

${ }^{2}$ Junior Resident, Department of Cheat and TB, Government Medical College, Amritsar, Punjab 143001, India

DOI: $10.36347 /$ sjams.2020.v08i07.027

| Received: 15.07.2020 | Accepted: 23.07.2020 | Published: 28.07.2020

*Corresponding author: Ritu Dadra

Abstract

Original Research Article

This is an observational prospective study, which included 200 microbiologically confirmed cases of tuberculosis (TB) which came to outpatient department or got admitted in the wards. Clinico-diagnostic profile of these cases was determined. The collected data were documented, compiled, tabulated and statistically analysed to reach a valid conclusion. Out of 200 microbiologically confirmed cases of TB, 25\% of the cases were in the age group of 21-30 years, $147(73.5 \%)$ were drug susceptible (DS) and 53 (26.5\%) were drug resistant (DR). Amongst DR-TB cases, 21 $(18.7 \%)$ were new and $32(36.4 \%)$ were previously treated cases. Rifampicin mono-resistance was higher in both new $(47.6 \%)$ and previously treated $(37.5 \%)$ drug resistance cases. The majority of the patients with DR-TB had previous history of ATT i.e., ( $\mathrm{p}$ value $<0.5$ ). Risk factors associated with DR-TB are female gender and previous history of Anti-tubercular therapy (ATT) (p value <.05).

Keywords: Drug resistant Tuberculosis; Mycobacterium tuberculosis; Anti-tubercular therapy; Rifampicin.

Copyright @ 2020: This is an open-access article distributed under the terms of the Creative Commons Attribution license which permits unrestricted use, distribution, and reproduction in any medium for non-commercial use (NonCommercial, or CC-BY-NC) provided the original author and source are credited.

\section{INTRODUCTION}

Tuberculosis remains a worldwide public health problem despite the fact that the causative organism was discovered more than 100 years ago [1]. Emergence of drug resistance has further worsened the situation and has become a significant health problem world over creating an obstacle to effective tuberculosis control as the treatment is much more toxic and much more expensive than the one of patients with sensitive organisms [2].

The burden of DR-TB is of major interest and concern at global, regional and country levels. In 2018, there were approximately half a million (range, 417 000-556 000) new cases of rifampicin-resistant TB (RR-TB) of which $78 \%$ had MDR-TB. The three countries with the largest share of the global burden were India $(27 \%)$, China $(14 \%)$ and the Russian Federation (9\%). Globally, $3.4 \%$ of new TB cases and $18 \%$ of previously treated cases had MDR/RR-TB, with the highest proportions $(>50 \%$ in previously treated cases) in countries of the former Soviet Union. Currently, the WHO estimated incidence of R and MDR TB in India is estimated to be around 130 000. This translates to around 9.6 patients per 100000 population annually as per the Global TB Report, 2019 [3].
MDR-TB mirrors the functional state and efficacy of tuberculosis control programs and realistic attitude of the community towards the implementation of such programs in the country [4]. Good treatment is pre-requisite to the prevention of emergence of resistance. India has one-fifth of the world's multidrug resistant tuberculosis burden- the largest number for any country globally after China [6]. MDR-TB is a manmade phenomenon- Poor treatment, poor drugs and poor adherence lead to the development of MDR-TB [5].

Most of the studies found that cavity was the most common radiological lesion of multidrug resistant tuberculosis patients on chest X-rays. The limited drug penetration into the cavity that harbor large mycobacterial load and a greater number of Acid fast bacilli (AFB) in moderately advanced or far advanced disease is believed to contribute to drug resistance [6].

The treatment of DR-TB is extremely challenging owing to the complexity of chemotherapy regimens, the toxicity of alternative drugs, and the high cost of these drugs. Therefore, it is particularly important to identify the risk factors associated with DR-TB. Judicious use of drugs, supervised individualized treatment, focused clinical, radiological and bacteriological follow up, use of surgery at the 
appropriate juncture are key factors in the successful management of these patients.

This research is believed to contribute to the pattern of drug resistance and to identify the potential risk factors for DR-TB, so that the management of patients will also be strengthened through preventing these factors, alongside patient treatment which will have a positive impact on the successful treatment outcome, and decrease the burden of the disease as a whole.

\section{Materials ANd Methods}

This study was carried out in the Department of Chest and Tuberculosis, Government Medical College, Amritsar. This is an observational prospective study, which included 200 microbiologically confirmed cases of tuberculosis, which came to outpatient department or got admitted in the wards over a period of 18 months. The approval of institutional thesis and ethics committee was taken before the start of the study. Participants who met the inclusion criteria was recruited after giving information regarding the study in their vernacular language and written informed consent was obtained.

\section{Inclusion Criteria}

- All consenting new and previously treated microbiologically confirmed cases of tuberculosis of any age and gender visiting chest and TB hospital Amritsar was included in the study.

\section{Exclusion Criteria}

1. Patients with tuberculosis not confirmed by microbiology.

2. Critically ill / moribund patients.

3. Patients not willing to participate in the study.

4. Patient not capable of giving consent (psychiatric patient).

All diagnosed Patients of MDR-TB referred to DR-TB-Centre were admitted for Cat-IV treatment under the programmatic management of drug- resistant tuberculosis (PMDT)- revised national tuberculosis programme (RNTCP) in directly observed treatment, short course (DOTS) Plus ward, department of TB \& Chest, Government Medical College, Amritsar for pretreatment evaluation for a minimum duration of seven days. The protocol was clearly explained to the patient/care provider before enrolment and informed consent was taken from each patient. A pre-structured proforma will be filled in those cases which are included in the study.

1. Detailed clinical history and clinical examination.

2. Complete blood count.

3. Blood sugar to screen for diabetes mellitus.

4. Liver function test.
5. Blood urea and serum creatinine to assess the Kidney function test.

6. Urine examination- routine and microscopic.

7. Pregnancy test (for all women in child bearing age group).

8. Chest X-ray.

9. All DR-TB cases will be offered a referral for human immunodeficiency virus (HIV) counselling and testing at the nearest center if the HIV status is not known or the HIV test is found negative with results more than 6 months old. If the patient is HIV positive, he /she will be referred to anti-retroviral therapy (ART) centre (if not on ART).

Demographic characteristics, socioeconomic status, complete detailed clinical history regarding total duration of illness, smoking history, drug/alcohol abuse, mental illness, diabetic history, previous anti tuberculosis therapy, family history of anti- tuberculosis therapy and any contact with tuberculosis patients will be taken from the patients. All sputum positive patients will be subjected to chest radiograph.

The collected data was documented, compiled, tabulated and statistically analysed to reach a valid conclusion.

\section{RESULTS}

In our study, out of 200 patients taken for the study maximum number of patients were present in the age group of 21-30 years i.e. $25 \%$. Mean age was 40.15 years. There was an overall male preponderance in the study with $60 \%$ being males, $40 \%$ being females. 110 $(55 \%)$ patients were from urban population and 90 (45\%) patients belonged to rural areas.

Most of the patients were Laborers 63 (31.5\%). The most common symptom was cough with expectoration which was present in 185 (92.5\%) of cases followed by fever, which was present in 155 $(77.5 \%)$ cases. Pallor, clubbing and lymphadenopathy were present in $155(77.5 \%), 37(18.5 \%), 46(23 \%)$ of the patients respectively. $33(16.5 \%)$ patients were Diabetic, $6(3 \%)$ were diagnosed cases of HIV. Most common addiction was smoking present in 79 (39.5\%) of study subjects, followed by alcohol which was present in $72(36 \%)$ of the cases. $87(43.5 \%)$ patients were having a previous history of ATT, $113(56.5 \%)$ patients were not having any previous history of ATT. History of exposure was present in $50(34 \%)$ cases in drug susceptible cases and $16(30.1 \%)$ in drug resistant cases.

$147(73.5 \%)$ were drug susceptible and 53 $(26.5 \%)$ were drug resistant. Total no. of new and previously treated cases were $91(82.1 \%)$ and 56 $(63.6 \%)$ in drug susceptible and $21(18.7 \%)$ and 32 $(36.4 \%)$ in drug resistant cases. 
Out of 21 drug resistant new cases, $10(47.6 \%)$ patients were Rifampicin mono resistant, 5 (23.8\%) were multi-drug resistant, $4(19 \%)$ were isoniazid resistant and $2(9.5 \%)$ were rifampicin plus fluoroquinolone resistant. Among 32 previously treated drug resistant cases, $12(37.5 \%)$ patients were Rifampicin mono resistant, $9(28.1 \%)$ were multi-drug resistant, $5(15.6 \%)$ were isoniazid mono resistant 4 $(12.5 \%)$ were rifampicin plus fluoroquinolone resistant, $1(3.1 \%)$ each of extensive drug resistant and isoniazid plus fluoroquinolone resistant ( $\mathrm{p}$ value $>.05$ which is not significant).

Majority of the patients $83(56.4 \%)$ has unilateral disease in drug susceptible cases and bilateral disease $23(43.4 \%)$ in drug resistant cases. Among drug susceptible cases, $37(25.1 \%)$ patients were having minimal disease $53(36 \%)$ and $48(32.6 \%)$ patients were having moderate and far advanced disease respectively. Among drug resistant cases, 5 (9.4\%) were having minimal disease, $8(15.1 \%)$ and $38(71.7 \%)$ patients were having moderate and far advanced disease. Risk factors associated with drug resistance TB are female gender and previous history of ATT ( $p$ value <.05) while other factors such as age less than 40 years, contact history, HIV status, smoking and alcoholism are not associated with Drug resistance TB.

\section{DiSCUSSION}

There were total of 200 microbiologically confirmed cases of TB, which included 194 sputum positive, 1 broncho-alveolar lavage (BAL) and 5 others (pleural fluid, pleural pus, fine needle aspirate) who were detected through smear microscopy and molecular methods i.e. line probe assay (LPA) or cartridge based nucleic acid amplification (CBNAAT). We evaluated patterns of drug resistance in these cases and risk factors associated with drug resistance.

There were 120 males and 80 were females in our study (Table-1) Peter et al., [7] and Fandinho et al., [8] have also reported male to female ratio of 1.8:1 and 1.6:1 respectively.This may be due to our male dominating society in which males have more access to health facilities as compared to female. Out of 200 patients, $110(55 \%)$ patients were from urban population and 90 (45\%) patients belonged to rural areas. Study by Kliiman et al., also showed that $61.7 \%$ patients were from urban areas and $38.3 \%$ patients were from rural areas [9]. Among the urban population narrow living spaces, poorly ventilated houses, overcrowding and unhealthy food habits could be cause of tuberculosis.

In our study most of the patients were laborers $(31.5 \%)$. Laborers belong to lower socioeconomic status and as we know tuberculosis affects especially those from lower socioeconomic background. Poverty could be a major predisposing factor, ranging in them from poor overall hygiene, poor living conditions, poor nutrition and immune status.

Among 200 patients taken in the study, the most common symptom was cough with expectoration present in $92.5 \%$ of cases followed by fever in $77.5 \%$. Breathlessness was present in $77.5 \%$. Loss of appetite was present in $66 \%$ and weight loss was present in $45 \%$ of patients and hemoptysis in $10 \%$ of cases. Among drug susceptible cases history of contact with pulmonary TB case was present in $44(29.9 \%)$ and MDR-TB case in $6(4.1 \%)$ of the study subjects. Among drug resistant cases history of contact with pulmonary TB case was present in $12(22.6 \%)$ and MDR-TB case in $4(7.5 \%)$ of the study subjects.

Most common drug addiction in our study was smoking present in $79(39.5 \%)$ of study subjects, followed by alcohol, which was present in 72 (36\%) of the cases. Study done by Mishra et al., [10] also reported that smoking was commonest drug addiction followed by alcohol. HIV positive patients in our study were $6(3 \%)$ and the rest were HIV negative. 33 $(16.5 \%)$ were diabetic and $167(82.5 \%)$ were non diabetic. Diabetes was commonest comorbidity. Among other comorbidities after diabetes COPD was commonest comorbidity. COPD present in $14(7 \%)$ which was smoking related, followed by Hypertension in $10(5 \%)$, Hypothyroidism and depression in $3(1.5 \%)$.

Out of 200 patients $147(73.5 \%)$ were drug susceptible and $53(26.5 \%)$ were drug resistant in our study. Total no. of new and previously treated cases were $91(82.1 \%)$ and $56(62.5 \%)$ in drug susceptible and $21(18.7 \%)$ and $32(36.4 \%)$ in drug resistant cases. Out of 21 drug resistant new cases, $10(47.6 \%)$ patients were Rifampicin mono resistant, 5 (23.8\%) were multidrug resistant, $4(19 \%)$ were isoniazid resistant and 2 (9.5\%) were rifampicin plus fluoroquinolone resistant. Among 32 previously treated drug resistant cases 12 (37.5\%) patients were Rifampicin mono resistant, 9 $(28.1 \%)$ were multi-drug resistant, 5 (15.6\%) were isoniazid mono-resistant $4(12.5 \%)$ were rifampicin plus fluoroquinolone resistant, $1(3.1 \%)$ each of extensive drug resistant and isoniazid plus fluoroquinolone resistant (Table-2).

Findings similar to our study were reported by Dr. Nirmalya Manna, $53.7 \%$ patients were found to be resistant to Rifampicin only, $46.3 \%$ to both Rifampicin and Isoniazid [11]. In our study, out of 53 drug resistant cases majority $32(60.4 \%)$ cases have previous history of ATT (p value <.05). A study done by Sudhakar W. more reported out of 86 cases, the majority of the patients with drug-resistant TB had acquired drug resistance, i.e., $66(68.75 \%)$ [12].

Our study also showed that females were affected more than males in MDR-TB Cases ( $\mathrm{p}$ value $<.05)$. The reasons for the association between female 
and MDR-TB might be related to the fact that women spend more time caring their family members with MDR- TB both in households and healthcare settings. Previous history of treatment was present in 55 (37.4\%) cases in drug susceptible and $32(60.4 \%)$ in drug resistant cases ( $p$ value $<.05$ which is statistically significant). Risk factors associated with drug resistance TB are female gender and previous history of ATT (p value <.05) while other factors such as age less than 40 years, contact history, HIV status, smoking and alcoholism are not associated with Drug resistance TB (Table-3).

The majority of the patients $83(56.4 \%)$ has unilateral disease in drug susceptible cases and bilateral disease $23(43.4 \%)$ in drug resistant cases. Among drug susceptible cases, $37(25.1 \%)$ patients were having minimal disease $53(36 \%)$ and $48(32.6 \%)$ patients were having moderate and far advanced disease respectively. Among drug resistant cases, $5(9.4 \%)$ were having a minimal disease, $8(15.1 \%)$ and $38(71.7 \%)$ patients were having moderate and far advanced disease. Another retrospective cross- sectional study done by Icksan et al., [13] which compares chest $\mathrm{X}$ - ray findings of two groups of patients, involving $183 \mathrm{DS}-\mathrm{TB}$ patients and 183 MDR- TB patients. MDR- TB group have $177(96 \%)$ patients with large lesions, $6(4 \%)$ with medium lesions, and no small lesions. DS - TB group have $55(30 \%)$ patients with small lesions, $78(43 \%)$ with medium lesions, and $50(27 \%)$ with large lesions.

\section{CONCLUSION}

The prevalence of drug resistance was found to be high in previously treated patients as compared to new cases. Risk factors associated with DR-TB are female gender and previous history of ATT ( $p$ value $<.05)$ Immediate therapeutics and more surveillance are necessary to combat the threat of DR-TB. Knowledge of drug resistance patterns for new and previously treated cases and risk factors associated with drug resistance are critical for effective control of MDR-TB in different regions of the country. Prompt and accurate detection of drug resistance will be critical to timely initiation of treatment hence prevent further transmission and allows selection of a drug regimen to which the infecting strain is susceptible.

\section{REFERENCES}

1. Park, K. Tuberculosis. In: Park's Textbook of Preventive and Social Medicine, 20th Edition. Jabalpur; M/S Banarsidas Bhanot, 2009.

2. Mahmoudi A, Iseman MD. Pitfalls in the care of patients with tuberculosis: common errors and their association with the acquisition of drug resistance. Jama. 1993 Jul 7;270(1):65-8.

3. World Health Organization. Global Tuberculosis Report, 2019. Available from: https://apps.who.int/iris/bitstream/handle/10665/32 9368/9789241565714-eng.pdf?ua=1.

4. Guideline for PMDT in India 2017-1.pdf. Available from https://pdfs.semanticscholar.org/17ec/8a94c854e0 137e6800d973f6dbf167ff0d4f.pdf.

5. Caminero JA. A tuberculosis guide for specialist physicians. Paris: International Union Against Tuberculosis and Lung Disease. 2004 Sep;79.

6. The role of BCG vaccine in the prevention and control of tuberculosis in the United States. A joint statement by the Advisory Council for the Elimination of Tuberculosis and the Advisory Committee on Immunization Practices. MMWR Recomm Rep Morb Mortal Wkly Rep Recomm Rep. 1996 Apr 26;45(RR-4):1-18

7. Eriki PR, Okwera A, Aisu T, Morrissey AB, Ellner JJ, Daniel TM. The Influence of Human Immunodeficiency Virus Infection on Tuberculosis in Kampala, Uganda1-3. Am Rev Respir Dis. 1991;143:185-7.

8. Fandinho FC, Kritski AL, Hofer C, Conde Jr H, Ferreira RM, Silva MG, et al. Drug resistance patterns among hospitalized tuberculous patients in Rio de Janeiro, Brazil, 1993-1994. Memórias do Instituto Oswaldo Cruz. 1999 Jul;94(4):543-7.

9. Kliiman K, Altraja A. Predictors of poor treatment outcome in highly drug-resistant pulmonary tuberculosis. European Respiratory Journal. 2009 Jan 22

10. Mishra VK, Gupt P, Pachar P, Jangir SK, Gupta RC, Gour N. Original Research Article A Study to assess the profile of multidrug-resistant tuberculosis (MDR-TB) in tertiary care hospital setting.

11. Manna N, Giri K, Mundle M. Drug resistance pattern, related sociodemographic factors and preventive practices among MDR-TB patients: An experience from a tertiary care setting. Journal of Dental and Medical Sciences. 2014;13(9):16-21

12. More SW, Parande MA, Kamble SW, Kamble MS. Profile of drug-resistant tuberculosis in Western Maharashtra. Journal of family medicine and primary care. 2017 Jan;6(1):29.

13. Icksan AG, Napitupulu MR, Nawas MA, Nurwidya F. Chest X-ray findings comparison between multi-drug-resistant tuberculosis and drug-sensitive tuberculosis. Journal of natural science, biology, and medicine. 2018 Jan;9(1):42. 